PROCEEDINGS OF THE

AMERICAN MATHEMATICAL SOCIETY

Volume 129, Number 2, Pages 525-530

S 0002-9939(00)05549-0

Article electronically published on September 18, 2000

\title{
THE SIZE OF THE DINI SUBDIFFERENTIAL
}

\author{
JÖ̈L BENOIST
}

(Communicated by David R. Larson)

\begin{abstract}
Given a lower semicontinuous function $f: \mathbb{R}^{h} \rightarrow \mathbb{R} \cup\{+\infty\}$, we prove that the points of $\mathbb{R}^{h}$, where the lower Dini subdifferential contains more than one element, lie in a countable union of sets which are isomorphic to graphs of some Lipschitzian functions defined on $\mathbb{R}^{h-1}$. Consequently, the set of all these points has a null Lebesgue measure.
\end{abstract}

\section{INTRODUCTION}

Let $f: \mathbb{R}^{h} \rightarrow \mathbb{R} \cup\{+\infty\}$ be any lower semicontinuous function. An important and often-featured notion of generalized (directional) derivative is the lower Dini derivative, which, for $x$ in the domain of $f$, is defined by

$$
d^{-} f(x ; d)=\liminf _{u \rightarrow d, t \rightarrow 0^{+}} \frac{f(x+t u)-f(x)}{t} .
$$

We associate to it the lower Dini subdifferential of $f$ at a point $x$, in the domain of $f$, defined by

$$
\partial^{-} f(x)=\left\{\xi \in \mathbb{R}^{h}:\langle\xi, d\rangle \leq d^{-} f(x, d), \forall d \in \mathbb{R}^{h}\right\} .
$$

Let us put $\partial^{-} f(x)=\emptyset$ if $f(x)=+\infty$. The lower Dini subdifferential is a closed convex set, which can be described as follows:

$$
\partial^{-} f(x)=\left\{\xi \in \mathbb{R}^{h}: \liminf _{y \rightarrow x} \frac{f(y)-f(x)-\langle\xi, y-x\rangle}{\|y-x\|} \geq 0\right\} .
$$

We refer to the book [1] for the above characterization and other classical properties of this subdifferential. This paper deals with the "size" of the two following subsets which are included in the domain of the lower Dini subdifferential of $f$ at $x$ :

$$
\begin{gathered}
D(f)=\left\{x \in \mathbb{R}^{h}: \text { the interior of } \partial^{-} f(x) \text { is nonempty }\right\} \\
\tilde{D}(f)=\left\{x \in \mathbb{R}^{h}: \partial^{-} f(x) \text { contains at least two elements }\right\} .
\end{gathered}
$$

Let us notice that the inclusion $D(f) \subset \tilde{D}(f)$ is always true; moreover if $h=1$, we have $D(f)=\tilde{D}(f)$.

Received by the editors October 7, 1998 and, in revised form, May 3, 1999.

2000 Mathematics Subject Classification. Primary 26A16, 26A24.

Key words and phrases. Lower semicontinuous function, Dini subdifferential, proximal subdifferential, countability, null Lebesgue measure set. 


\section{Countability of the set $D(f)$}

In this section, we focus on the cardinality of the set $D(f)$. As shown in the following example, the set $D(f)$ is in general not finite. Let us consider the function $f$ whose epigraph is the convex hull of the set

$$
\left\{\left(n, n^{2}\right): n \in \mathbb{Z}\right\} \text {. }
$$

In this case, $D(f)=\mathbb{N}$ and so $D(f)$ is infinite countable. The first result shows that this property is still true for any lower semicontinuous function.

Proposition 2.1. For any lower semicontinuous function $f$, the set $D(f)$ is at most countable.

Proof. Let us choose a dense sequence $\left(v_{p}\right)_{p \geq 1}$ in $\mathbb{R}^{h}$ dense in $\mathbb{R}^{h}$ and let us define, for $\omega=(p, n, \ell) \in\left(\mathbb{N}^{\star}\right)^{3}$, the subset $D_{\omega}$ constituted of elements $x$ of $\mathbb{R}^{h}$ such that $f(x)$ is real and such that, for all $\xi \in \bar{B}\left(v_{p}, \frac{1}{n}\right)$ and for all $y \in \bar{B}\left(x, \frac{1}{\ell}\right)$,

$$
f(y) \geq f(x)+\langle\xi, y-x\rangle-\frac{1}{3 n}\|y-x\| .
$$

Step 1. Let us prove the inclusion $D(f) \subset \bigcup_{\omega \in\left(\mathbb{N}^{*}\right)^{3}} D_{\omega}$.

Let $x \in D(f)$; from the definition of the set $D(f), f(x)$ is real. Since the interior of the subdifferential $\partial^{-} f(x)$ is nonempty and since the sequence $\left(v_{p}\right)_{p \geq 1}$ is dense in $\mathbb{R}^{h}$, there exist an integer $p \geq 1$ and $\epsilon>0$ such that

$$
\bar{B}\left(v_{p}, \epsilon\right) \subset \partial^{-} f(x) .
$$

Let $\left(e_{1}, \cdots, e_{h}\right)$ be the canonical basis of $\mathbb{R}^{h}$; for $i \in\{1, \cdots, h\}$, we put $e_{h+i}=-e_{i}$. According to relation (11), for every $i \in\{1, \cdots, 2 h\}$, we have $v_{p}+\epsilon e_{i} \in \partial^{-} f(x)$, whence there exists a constant $r_{i}>0$ such that

$$
f(y) \geq f(x)+\left\langle v_{p}+\epsilon e_{i}, y-x\right\rangle-\frac{1}{3 n}\|y-x\|,
$$

for all $y \in \bar{B}\left(x, r_{i}\right)$. Let us choose an integer $\ell \geq 1$ such that

$$
\frac{1}{\ell} \leq \min \left\{r_{i}: i \in\{1, \cdots, 2 h\}\right\} \text {. }
$$

By convex combinations of inequalities (2) $(i=1, \cdots, 2 h)$, we obtain:

$$
f(y) \geq f(x)+\left\langle v_{p}+u, y-x\right\rangle-\frac{1}{3 n}\|y-x\|,
$$

for all $y \in \bar{B}\left(x, \frac{1}{\ell}\right)$ and for all $u \in \operatorname{co}\left\{\epsilon e_{1}, \cdots, \epsilon e_{2 h}\right\}$. It is well-known that the null vector of $\mathbb{R}^{h}$ belongs to the interior of $\operatorname{co}\left\{\epsilon e_{1}, \cdots, \epsilon e_{2 h}\right\}$ and consequently, we can find an integer $n \geq 1$ such that

$$
\bar{B}\left(0, \frac{1}{n}\right) \subset \operatorname{co}\left\{\epsilon e_{1}, \cdots, \epsilon e_{2 h}\right\} .
$$

Hence inequality (3) is in particular valid, for all $y \in \bar{B}\left(x, \frac{1}{\ell}\right)$ and for all $u \in$ $\bar{B}\left(0, \frac{1}{n}\right)$. Then we conclude that $x \in D_{(p, n, \ell)}$ and the result is proved.

Step 2. Let us show that, for all $\omega=(p, n, \ell) \in\left(\mathbb{N}^{\star}\right)^{3}$ and for all elements $x_{1}, x_{2}$ of $D_{\omega}$, the condition $x_{1} \neq x_{2}$ implies $\left\|x_{2}-x_{1}\right\|>\frac{1}{\ell}$.

Suppose to the contrary that there exist $\omega=(p, n, \ell)$ in $\left(\mathbb{N}^{\star}\right)^{3}$ and two distinct elements $x_{1}, x_{2}$ in $D_{\omega}$ such that:

$$
\left\|x_{2}-x_{1}\right\| \leq \frac{1}{\ell}
$$


On one hand, since $x_{1} \in D_{\omega}$, by choosing $\xi=v_{p}+\frac{1}{n} \frac{x_{2}-x_{1}}{\left\|x_{2}-x_{1}\right\|}$, we obtain from step 1 :

$$
f\left(x_{2}\right) \geq f\left(x_{1}\right)+\left\langle v_{p}, x_{2}-x_{1}\right\rangle+\frac{2}{3 n}\left\|x_{2}-x_{1}\right\| .
$$

On the other hand, since $x_{2} \in D_{\omega}$, by choosing $\xi=v_{p}$, we obtain from step 1:

$$
f\left(x_{1}\right) \geq f\left(x_{2}\right)+\left\langle v_{p}, x_{1}-x_{2}\right\rangle-\frac{1}{3 n}\left\|x_{1}-x_{2}\right\| .
$$

Adding these two last inequalities, we conclude

$$
0 \geq \frac{1}{3 n}\left\|x_{1}-x_{2}\right\|,
$$

or equivalently $x_{1}=x_{2}$, which is impossible.

Step 3. Let us now conclude.

From step 2, for all $\omega \in\left(\mathbb{N}^{\star}\right)^{3}$, the set $D_{\omega}$ is discrete in $\mathbb{R}^{h}$ and, in particular, it is countable. Then, the inclusion proved in the step 1 allows us to confirm that the set $D(f)$ is also countable.

As we shall see in the next section, the structure of the set $\tilde{D}(f)$ is more complicated.

\section{The structure of the Set $\tilde{D}(f)$}

Henceforward we assume that $h \geq 2$. Let us remark that, for $h=2$ and $f\left(x_{1}, x_{2}\right)=\left|x_{1}\right|$, we have $\tilde{D}(f)=\{0\} \times \mathbb{R}$. In this example $\tilde{D}(f)$ is not countable, but it has a null Lebesgue measure. More generally, if $f$ is a Lipschitzian 1 function on $\mathbb{R}^{h}$, Rademacher's Theorem states that $f$ is differentiable at almost every point of $\mathbb{R}^{h}$ and in particular the set $\tilde{D}(f)$ has a null Lebesgue measure. Theorem 3.3 shows that this result is valid even if the Lipschitz property fails.

First, we introduce the following definition:

Definition 3.1. We say that a subset $X$ of $\mathbb{R}^{h}$ is Lipschitzian if there exist a linear isomorphism $\phi: \mathbb{R}^{h} \rightarrow \mathbb{R}^{h}$ and a Lipschitzian function $g: \mathbb{R}^{h-1} \rightarrow \mathbb{R}$ satisfying the equality

$$
X=\phi(\operatorname{Gr}(g)) \text {, }
$$

where $\operatorname{Gr}(g)$ denotes the graph of the function $g$.

According to Fubini's Theorem, the graph of a Lipschitzian function has a null Lebesgue measure; hence we have the following property.

Proposition 3.2. Any Lipschitzian set in $\mathbb{R}^{h}$ has a null Lebesgue measure.

We can now establish the main result of this paper.

Theorem 3.3. If $f$ is a lower semicontinuous function, the set $\tilde{D}(f)$ is included in a countable union of Lipschitzian sets of $\mathbb{R}^{h}$. In particular, $\tilde{D}(f)$ has a null Lebesgue measure.

\footnotetext{
${ }^{1}$ I.e., there exists $K>0$ such that $|f(x)-f(y)| \leq K|| x-y \|$ for all $x, y$ in $\mathbb{R}^{h}$.
} 
Proof. Let us choose a sequence $\left(v_{p}\right)_{p \geq 1}$ in $\mathbb{R}^{h} \backslash\{0\}$ which is dense in $\mathbb{R}^{h}$ and let us define, for $\omega=(n, p, q, \ell) \in\left(\mathbb{N}^{\star}\right)^{4}$, the compact $\tilde{D}_{\omega}$ constituted of elements $x$ belonging to the closed ball $\bar{B}(0, n)$ such that $f(x) \leq n$ and such that there exist $d \in \bar{B}\left(v_{p}, \frac{1}{n}\right) \cap\left[\mathbb{R}^{h} \backslash B\left(0, \frac{9}{n}\right)\right]$ and $\xi \in \bar{B}\left(v_{q}, \frac{1}{2 n}\right)$ satisfying:

$$
\forall y \in B\left(x, \frac{1}{\ell}\right), f(y) \geq f(x)+\langle\xi, y-x\rangle+|\langle d, y-x\rangle|-\frac{1}{2 n}\|y-x\| .
$$

Step 1. Let us prove the inclusion $\tilde{D}(f) \subset \bigcup_{\omega \in \Omega} \tilde{D}_{\omega}$, where $\Omega=\left\{\omega \in\left(\mathbb{N}^{\star}\right)^{4}\right.$ : $\left.\tilde{D}_{\omega} \neq \emptyset\right\}$.

Let $x \in \tilde{D}(f)$; there exist two different elements $\xi_{1}, \xi_{2}$ belonging to $\partial^{-} f(x)$. We can write $\xi_{1}=\xi+d$ and $\xi_{2}=\xi-d$, with $d \neq 0$; let us choose an integer $n \geq 1$ such that $f(x) \leq n,\|x\| \leq n$ and $\|d\| \geq \frac{9}{n}$. On one hand, since the sequence $\left(v_{p}\right)_{p \geq 1}$ is dense, we can find two integers $p \geq 1$ and $q \geq 1$ such that $v_{p} \in \bar{B}\left(d, \frac{1}{n}\right)$ and $v_{q} \in B\left(\xi, \frac{1}{2 n}\right)$. On the other hand, from the characterization of lower Dini subdifferential, there exists an integer $\ell \geq 1$ such that, for $\epsilon \in\{1,-1\}$,

$$
\forall y \in B\left(0, \frac{1}{\ell}\right), f(y) \geq f(x)+\langle\xi, y-x\rangle+\epsilon\langle d, y-x\rangle-\frac{1}{2 n}\|y-x\| .
$$

And so, the asserted inclusion holds.

Step 2. Let us fix $\omega=(n, p, q, \ell) \in \Omega$ and let us prove that, for all $x \in \tilde{D}_{\omega}$,

$$
\left(\{x\}+\left[B\left(0, \frac{1}{\ell}\right) \cap K\left(v_{p}\right)\right]\right) \backslash\{x\} \subset \mathbb{R}^{h} \backslash \tilde{D}_{\omega},
$$

where $K\left(v_{p}\right):=\left\{k \in \mathbb{R}^{h}:\left\langle k, v_{p}\right\rangle \geq \frac{1}{2}\|k\|\left\|v_{p}\right\|\right\}$ denotes the closed convex cone of vectors $k$ such that the (radian) measure of the angle between $k$ and $v_{p}$ is less than $\arccos \frac{1}{2}\left(\right.$ remark that $\left.v_{p} \neq 0\right)$.

Let $y$ be an element of $\tilde{D}_{\omega}$ such that $\|y-x\|<\frac{1}{\ell}$ and

$$
\left\langle y-x, v_{p}\right\rangle \geq \frac{1}{2}\|y-x\|\left\|v_{p}\right\| .
$$

We must verify that $y=x$. On one hand, since $x \in \tilde{D}_{\omega}$, there exist $d_{x} \in \bar{B}\left(v_{p}, \frac{1}{n}\right) \cap$ $\left[\mathbb{R}^{h} \backslash B\left(0, \frac{9}{n}\right)\right]$ and $\xi_{x} \in \bar{B}\left(v_{q}, \frac{1}{2 n}\right)$ such that

$$
f(y) \geq f(x)+\left\langle\xi_{x}, y-x\right\rangle+\left|\left\langle d_{x}, y-x\right\rangle\right|-\frac{1}{2 n}\|y-x\| .
$$

But, from relation (4) and the Cauchy-Schwarz inequality, we deduce the following chain of inequalities:

$$
\begin{aligned}
\left|\left\langle d_{x}, y-x\right\rangle\right| & \geq\left|\left\langle v_{p}, y-x\right\rangle\right|-\left|\left\langle d_{x}-v_{p}, y-x\right\rangle\right| \\
& \geq \frac{1}{2}\|y-x\|\left\|v_{p}\right\|-\left\|d_{x}-v_{p}\right\|\|y-x\| .
\end{aligned}
$$

Hence, remarking that $\left\|d_{x}-v_{p}\right\| \leq \frac{1}{n}$ and $\left\|v_{p}\right\| \geq\left\|d_{x}\right\|-\left\|v_{p}-d_{x}\right\| \geq \frac{8}{n}$, we deduce

$$
\left|\left\langle d_{x}, y-x\right\rangle\right| \geq \frac{3}{n}\|y-x\| .
$$


On the other hand, since $y \in \tilde{D}_{\omega}$, there exists $\xi_{y} \in \bar{B}\left(v_{q}, \frac{1}{2 n}\right)$ such that

$$
f(x) \geq f(y)+\left\langle\xi_{y}, x-y\right\rangle-\frac{1}{2 n}\|x-y\| .
$$

Summing the inequalities (5), (6) and (7), we obtain

$$
\begin{aligned}
0 & \geq\left\langle\xi_{x}-\xi_{y}, y-x\right\rangle+\frac{2}{n}\|y-x\| \\
& \geq\left(\frac{2}{n}-\left\|\xi_{x}-\xi_{y}\right\|\right)\|y-x\| \\
& \geq \frac{1}{n}\|y-x\|
\end{aligned}
$$

and then $y=x$.

Step 3. Let us now conclude. By a compactness argument, if $\omega \in \Omega$, there exists a sequence $\left(x_{\omega}^{i}\right)_{i \in \mathbb{N}} \subset \tilde{D}_{\omega}$ taking a finite number of values such that $\tilde{D}_{\omega} \subset$ $\bigcup_{i \in \mathbb{N}} \bar{B}\left(x_{\omega}^{i}, \frac{1}{2 \ell}\right)$. Then, let us consider, for $i \in \mathbb{N}$, the compact sets $\tilde{D}_{\omega}^{i}=\tilde{D}_{\omega} \cap$ $\bar{B}\left(x_{\omega}^{i}, \frac{1}{2 \ell}\right)$ which satisfy the equality $\tilde{D}_{\omega}=\bigcup_{i \in \mathbb{N}} \tilde{D}_{\omega}^{i}$.

From step 1, it follows that $\tilde{D}(f) \subset \bigcup_{(\omega, i) \in \Omega \times \mathbb{N}} \tilde{D}_{\omega}^{i}$ and so it is sufficient to show that, for all $(\omega, i) \in \Omega \times \mathbb{N}, \tilde{D}_{\omega}^{i}$ is included in a Lipschitzian set. Setting $Y=\tilde{D}_{\omega}^{i}-K\left(v_{p}\right), Y$ is a proper closed subset of $\mathbb{R}^{\ell}$ and $Y-K\left(v_{p}\right) \subset Y$. The fact that the boundary of $Y$, denoted $\partial Y$, is Lipschitzian (see Definition 2.2) is a straighforward consequence of the following lemma which gives a standard criterion for a set to be Lipschitzian; see for example [3].

Lemma 3.4. Let $Y$ be a nonempty closed subset of $\mathbb{R}^{h}$ such that $Y \neq \mathbb{R}^{h}$ and $Y-K \subset Y$, where $K$ is a closed convex cone of $\mathbb{R}^{h}$ with a nonempty interior. Let $e_{h}$ be a unit vector belonging to the interior of $K$ and $\left(e_{1}, \cdots, e_{h-1}\right)$ an orthonormal set of the vector subspace $e_{h}^{\perp}=\left\{x \in \mathbb{R}^{h}:\left\langle x, e_{h}\right\rangle=0\right\}$. Then $g: \mathbb{R}^{h-1} \rightarrow \mathbb{R}$ defined by $\left(\lambda_{1}, \cdots, \lambda_{h-1}\right) \mapsto \sup \left\{t \in \mathbb{R}: \sum_{i=1}^{h-1} \lambda_{i} e_{i}+t e_{h} \in Y\right\}$ is a Lipschitzian function and $\partial Y=\phi(G r(g))$, where $\phi$ denotes the isomorphism from $\mathbb{R}^{h}$ to $\mathbb{R}^{h}$ defined by $\left(\lambda_{1}, \cdots, \lambda_{n}\right) \mapsto \sum_{i=1}^{h} \lambda_{i} e_{i}$

Our last task is to prove the inclusion $\tilde{D}_{\omega}^{i} \subset \partial Y$. If it is not true, there exist $b \in \tilde{D}_{\omega}^{i}$ and $\eta>0$ such that $\bar{B}(b, \eta) \subset Y$. In particular $b+k$ (with $k=\eta v_{p} /\left\|v_{p}\right\|$ ) can be written as $b^{\prime}-k^{\prime}$, where $b^{\prime} \in \tilde{D}_{\omega}^{i}$ and $k^{\prime} \in K\left(v_{p}\right)$. Since $K\left(v_{p}\right)$ is a convex cone, $k+k^{\prime} \in K\left(v_{p}\right)$. From equality $k+k^{\prime}=b^{\prime}-b$, we also have $k+k^{\prime} \in \bar{B}\left(0, \frac{1}{\ell}\right)$. Hence $b^{\prime}=b+\left(k+k^{\prime}\right) \in\{b\}+\left[\bar{B}\left(0, \frac{1}{\ell}\right) \cap K\left(v_{p}\right)\right]$ and, from step $2, b^{\prime}=b$. We conclude that $0 \neq k=-k^{\prime}$, which contradicts the fact that $K\left(v_{p}\right)$ is a pointed cone.

Remark 3.5. In the same manner, the definition of the sets $D(f)$ and $\tilde{D}(f)$ can be adapted to any subdifferential included in Dini's one. In particular we can apply these results to the proximal subdifferential (see [2], 4], [5], and [6]).

\section{REFERENCES}

[1] J.-P. Aubin and H. Frankowska, "Set-Valued Analysis", Birkhäuser, Basel, 1990. MR 91d:49001

[2] J. Benoist, Intégration du sous-différentiel proximal: un contre exemple, Can. J. Math., 50 (2), 1988, p. 242-265. CMP 98:13

[3] J.M. Bonnisseau and B. Cornet, Existence of equilibria when firms follow bounded losses pricing rule, Journal of Mathematical Economics, 17, 1988, p. 119-147. MR 91b:90051

[4] F.H. Clarke and R.M. Redheffer, The proximal subgradient and constancy, Canad. Math. Bull., 36, 1993, p. 30-32. MR 93m:28004 
[5] F.H. Clarke, R.J. Stern and P.R. Wolenski, Subgradient criteria for monotonicity, the Lipschitz condition, and convexity, Can. J. Math., 45, $\mathrm{n}^{0}$ 6, 1993, p. 1167-1183. MR 94j:49018

[6] R.T. Rockafellar, Proximal subgradients, marginal values, and augmented lagrangians in nonconvex optimization, Math. Op. Res., 6, 1981, p. 424-436. MR 83m:90088

Maître de Conférences, LACO, CNRS-ESA 6090, Université de Limoges, 87060 LimoGES, France

E-mail address: benoist@unilim.fr 